\title{
PROJECTION PROFILE BASED NUMBER PLATE LOCALIZATION AND RECOGNITION
}

\author{
Sandipan Chowdhury ${ }^{1}$, Arindam Das ${ }^{2}$, and Punitha $\mathrm{P}^{2}$ \\ ${ }^{1}$ Department of Computer Science and Engineering,Technology Campus \\ University of Calcutta, India \\ ${ }^{2}$ Imaging Tech Lab, HCL Technologies, India \\ sandipanchowdhury6egmail.com \\ arindam.d@hcl.com; drpunitha.s@hcl.com;
}

\begin{abstract}
This paper proposes algorithms to localize vehicle number plates from natural background images, to segment the characters from the localized number plates and to recognize the segmented characters. The reported system is tested on a dataset of 560 sample images captured with different background under various illuminations. The performance accuracy of the proposed system has been calculated at each stage, which is $97.1 \%, 95.4 \%$ and $95.72 \%$ for localisation \& extraction, character segmentation and character recognition respectively. The proposed method is also capable of localising and recognising multiple number plates in images.
\end{abstract}

\section{KEYWORDS}

Number plate localization, number Plate recognition, Character segmentation and recognition

\section{INTRODUCTION}

With overwhelming development of various computer vision techniques, a good number of applications have been employed in transport systems for varied purposes such as automatic toll tax collection, adding security measures in restricted areas, tracking in-out time of cars in parking lot and many more. Number plate recognition aiding the above purposes, also has a wide application in law enforcement and surveillance at traffic signals, speed limit junctions etc. Computer vision driven automatic number plate recognition systems are expected to automatically take photographs of vehicles and uniquely identify vehicles when they pass through certain points where these monitoring devices are installed.

Research findings in this domain can be dated back to 1970s, which is the result of the need to implement law enforcement and traffic control on transportation systems. The basic requirements of complete and accurate automation of this system, still keeps the research avenues in this area open even today. The ideal expectation for practical implementation and real-time usage is that the accuracy must be $100 \%$ and the computation complexity should be the possible minimum. A

Jan Zizka et al. (Eds) : CCSEIT, AIAP, DMDB, MoWiN, CoSIT, CRIS, SIGL, ICBB, CNSA-2016

pp. 185-200, 2016. @ CS \& IT-CSCP 2016

DOI : $10.5121 /$ csit.2016.60615 
few reasons which makes this a challenging task are the problems arising from natural/dynamic scene analysis; varying light conditions; different weather conditions; camera capturing limitations; Effects of distortion, blur and occlusion; language/scripts used; and those challenges raising with vehicle motions. In addition to these, some problems which cannot be addressed by computer vision solutions are worn out number plates which are not illuminated well during hours of darkness, dirty and broken etc. Most approaches therefore work only under restricted conditions such as fixed illumination, limited vehicle speed, designated routes, and stationary backgrounds.

This paper presents an algorithm to recognize number plates from natural scenes. The proposed method comprises of three main stages, localization of the number plate, character segmentation, and character recognition. Localisation stage includes binarization and noise elimination; locating the number plate region; extracting the number plates. An adaptive threshold based global binarization and locally applied Otsu's binarization are combined to obtain a more accurate binarization which retains the number plate and its characters intact. Localization \& extraction of the number plate follows projection profile based approach. This approach helps to identify the possible regions of number plates in the images. These regions are further examined to select number plate region for further processing or to discard. This technique helps to localize and extract multiple number plates present in a single image.

In character segmentation, projection profile technique, in addition to an approximation algorithm is used to segment each character from the number plate. For character recognition, Support Vector Machine (SVM) is used. The segmented characters are classified into 36 classes, 26 classes of alphabets and 10 classes of numerals. The performance of the system has been computed at each stage and is found to be around $97.1 \%, 95.4 \%$ and $95.72 \%$. The overall accuracy of the system is $92.68 \%$.

The remaining of the paper is organized as follows. A brief background of related work is given in Section II. The proposed algorithm is described in Section III. Details of database are given in Section IV, Experimental results and comparison study is presented in Section V. Discussion and conclusions are drawn in Section VI.

\section{RELATED WORK}

Salter in 1984 [1], presented the potential applications of automatic vehicle identification for vehicle weighing and classification. Dickinson and Waterfall [2], in the same conference presented a general discussion on the suitability of image/video processing to perform collection of data, automatic surveillance, automatic incident detection, vehicle tracking, and vehicle classification. Since, then many researchers [1] - [26] have worked towards addressing various challenges in Number plate recognition.

Anagnostopoulos et al. [3], gives a survey on the work carried out till 2007. The article categorizes and assesses numerous techniques developed for license plate recognition in still images or video sequences. Shan Du et al., [7], present a comprehensive review of the state-ofthe-art techniques for Automatic License Plate Recognition (ALPR) till 2013. The methods are categorized into different ALPR techniques according to the features the methods used at each stage, and compare them in terms of pros, cons, recognition accuracy, and processing speed. 
Suresh et al., [4], proposed a novel method to enhance license plate numbers of moving vehicles in real traffic videos. Clemens et al., [5] present full-featured license plate detection and recognition system implemented on an embedded DSP platform processing video streams in realtime. Shapiro et al., [6], proposed an inexpensive automatic solution for remote vehicle identification. The recognition scheme combined adaptive iterative threshold with a templatematching algorithm. In [8], Chang et al., has attempted to take care of some restricted working environment conditions. The technique use fuzzy disciplines to extract license plates from an input image, and neural network aims to identify the number present in a license plate.

Azad and Shayegh [9], use adaptive threshold to obtain binary images and then use edge detection and morphological operations to localize number plates. Hsu et al., [10] proposed edge clustering mechanism for number plate detection, maximally stable extreme region (MSER) detector for character segmentation and LDA based character recognition. Chaudhary and Chincore [11] proposed 8-neighbor connectivity analysis to localize multiple number plates in Indian road conditions. Li et al., [12] used MSER to detect candidate characters and designed conditional random field models and through belief propagation inference estimated the license plate location. Carballido et al., [13] proposed a template matching approach to recognize license plate digits in outdoor parking entrance vehicles. Wen et al [15] proposed a method for number plate recognition which uses Bernsen's algorithm to binaries the images and connected component analysis to localize number plate regions and finally SVM to recognize the characters. Comeli et al., [16] have proposed a complete solution to recognize number plates by localizing the number plate through maximum local contrast, enhancing the images through Gaussian filters and histogram stretching, detecting and correcting tilt and finally recognizing characters through template matching. Many researchers have worked on number plate recognition of multi-style and multi-nations. Shiying et al [14], proposed a decision tree based localization of multinational license plate. Jiao et al., [20] proposed a morphology driven method for multi-style, multinational license plate recognition. Thome et al., [21], used gradient density to localize number plates and used hierarchical neural network to perform character recognition through classification. Guo and Liu [22], proposed a self-learning and hybridized technique for license plate localization. AlGhaili et al., [23] proposed license plate detection through the study of vertical line structures in the image. Naito et al., [19] proposed a robust license plate recognition system capable of recognizing number plates of moving vehicles in outdoors using wide dynamic range cameras. Chang [17] also proposed a line detection and projection based method to localize number plates and normal factors to recognize characters. Wang and Liu [18] used morphological operators and connected component analysis to localize the number plates. Poon et al., [25] also used several greyscale morphological operators to localize number plates. Sirithinaphong and Chamnongthai [24], used projection profiles to localize number plates and then used back propagation neural network to classify the characters.

The existing license plate recognition systems address the problem of recognition through three sub-tasks viz., license plate localization, character segmentation and character recognition. Similarly, in the next section we present in detail the algorithm we propose to recognize Indian number plates in outdoor scenes.

\section{PROPOSED SYSTEM}

The proposed system comprises three main stages, localization, character segmentation \& normalization, and recognition. 


\subsection{Number Plate Localisation}

The localisation stage focuses on detecting the number plate region from the captured image, and comprises of sub-stages, binarization, Noise removal through filtering, and region cropping.

\subsection{Binarization}

Let $I$ be an RGB image (Fig-1).I is converted into 8-bit gray scale image (Fig-2) $G$ by using the standard weights set by NTSC (National Television System Committee) which is given below.

$$
G(u, v)=0.229 R+0.587 G+0.114 B
$$

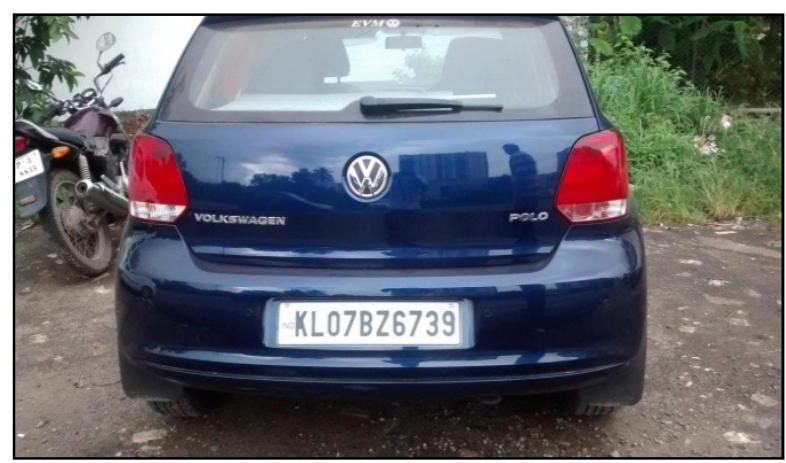

Figure 1. Input RGB image I

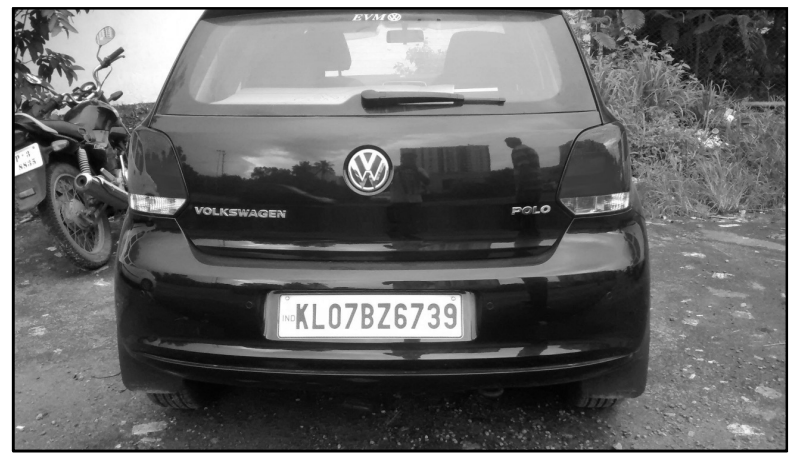

Figure 2. Gray scale image G of Fig.1

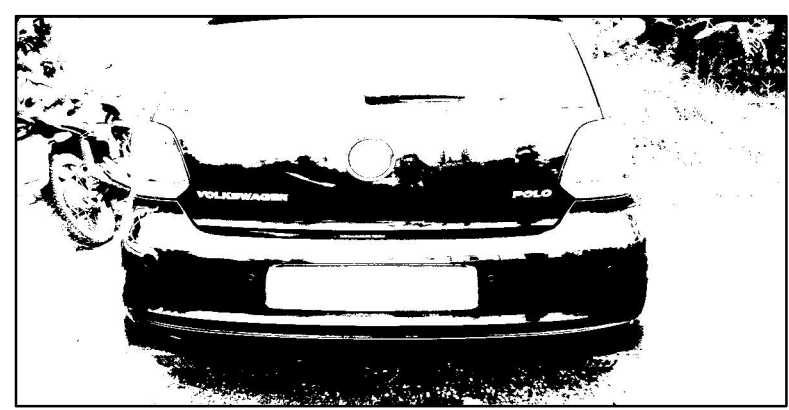

Figure 1. Binarized image B, using Otsu Global thresholding 
The gray scale image $G$ is then converted into binary image $B$, using well known Otsu's global threshold method (Fig-3). It was observed that while performing Otsu's method, although the image is pretty noiseless, the number plate gets eliminated due to global threshold. If the threshold is performed on local regions, the process can help to retain the number plate. To perform local threshold, $\mathrm{G}$ is divided into blocks of size $50 \times 50$ and Otsu's binarization is performed on each window of $50 \times 50$. Likewise window is shifted to acquire next $50 \times 50$ region of $G$ to perform the binarization iteratively on all regions. The output of applying Ostu'sbinarization on the image by considering local regions of 50x50 is shown in Fig-4. This local thresholding will introduce some noise along with retaining the number plate.

Global thresholding provided comparatively noiseless image with no or some part of number plate whereas local thresholding helped us to get the number plate but with more noise.

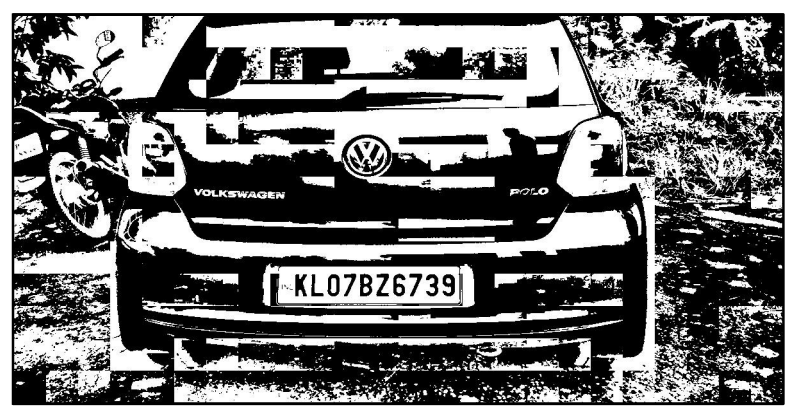

Figure 4. Binarized image C, using Otsu's method on local regions

\subsection{Connected Component Based Filtering}

The resultant image produced in binarization stage is noisy in nature. To eliminate the unwanted noise we perform a connected component based filtering. The connected components are examined if they are part of the number plate or not. Based on it verification the component is retained or removed.

It has been observed from the number plates that after binarization, the characters of the number plate are surrounded by good amount of white pixel region. This is one of the characteristics of number plates, having dark characters over lighter backgrounds. The rectangular structure of the plate contains darker characters over the lighter background which is a mandate. The characteristic feature of having white pixels around the characters of the number plate that can be seen in C, is used to localize the number plate. Fig-5 shows an example of 8-neighbor connected component.

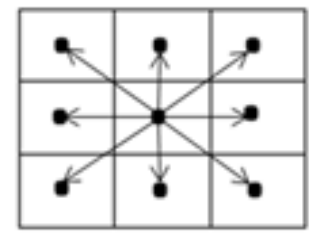

Figure 5. 8-Neighbor connectivity

To qualify a connected component as a possible number plate character we perform the following. 


\begin{tabular}{|c|c|c|c|c|c|}
\hline 0 & 1 & 1 & 1 & 1 & 1 \\
\hline 1 & 0 & 1 & 1 & 1 & 1 \\
\hline 1 & 0 & 1 & 0 & 0 & 1 \\
\hline 1 & 1 & 1 & 0 & 0 & 1 \\
\hline 1 & 1 & 1 & 1 & 1 & 1 \\
\hline 1 & 1 & 1 & 1 & 1 & 1 \\
\hline
\end{tabular}

Figure 2. 8 neighbour connected component analysis starting from the border pixel

The image $\mathrm{C}$ is scanned at the borders for a black pixel. Once a black pixel if found, this pixel is used as a seed to find all its 8 connected neighbours, i.e., while scanning the image if any border pixel $B_{p}$, is found to be black then we place a $\times 3$ window in such a way so that the pixel $B_{p}$ becomes the center of the $3 \times 3$ window. Let this $B_{p}$ be referred as the origin border pixel. Then the 8-neighbors which are also black and are connected to $\mathrm{B}_{\mathrm{p}}$ are noted. Any one of the neighbours which is connected to $\mathrm{B}_{\mathrm{p}}$ and is black in color becomes next center. The procedure is recursively repeated over all black pixels which are connected to $B_{p}$. All these pixels which form a region connected to the origin border pixel are eliminated, by changing them from black to white. For illustration we consider the below matrix, shown in Fig.6, where 0 represents the black pixel as the foreground and 1 represents the white pixel as the background.

In the above figure Fig-8 it is clearly observed that pixels $(0,0),(1,1),(2,1),(2,3),(2,4),(3,3)$ and $(3,4)$ are black pixels and among these pixels only $(0,0)$ is the border pixel. So pixel $(0,0)$ is made seed pixel. Now the 8 neighbour pixels of $(0,0)$ which are also black are found. The pixel at $(1,1)$ is found to be black, and again 8 connected neighbour analysis is done for $(1,1)$. The process recursively continues till no more new connections are found. For the border pixel $(0,0),(1,1)$ and $(2,1)$ are found as the 8 -connected neighbours. This region of three pixels is eliminated by replacing them by white pixels. This sequence is pictorially illustrated in Fig. 7.

\begin{tabular}{|c|c|c|c|c|c|}
\hline 1 & 1 & 1 & 1 & 1 & 1 \\
\hline 1 & 0 & 1 & 1 & 1 & 1 \\
\hline 1 & $\mathbf{1}$ & 1 & 0 & 0 & 1 \\
\hline 1 & 1 & 1 & 0 & 0 & 1 \\
\hline 1 & 1 & 1 & 1 & 1 & 1 \\
\hline 1 & 1 & 1 & 1 & 1 & 1 \\
\hline
\end{tabular}

(a)

\begin{tabular}{|l|r|r|r|r|r|}
\hline 1 & 1 & 1 & 1 & 1 & 1 \\
\hline 1 & 1 & 1 & 1 & 1 & 1 \\
\hline 1 & 0 & $\rightarrow 1$ & 0 & 0 & 1 \\
\hline 1 & 1 & 1 & 0 & 0 & 1 \\
\hline 1 & 1 & 1 & 1 & 1 & 1 \\
\hline 1 & 1 & 1 & 1 & 1 & 1 \\
\hline
\end{tabular}

(b) 


\begin{tabular}{|l|l|l|l|l|l|}
\hline 1 & 1 & 1 & 1 & 1 & 1 \\
\hline 1 & 1 & 1 & 1 & 1 & 1 \\
\hline 1 & 1 & 1 & 0 & 0 & 1 \\
\hline 1 & 1 & 1 & 0 & 0 & 1 \\
\hline 1 & 1 & 1 & 1 & 1 & 1 \\
\hline 1 & 1 & 1 & 1 & 1 & 1 \\
\hline
\end{tabular}

(c)

Figure 7. 8-neighbor connected component analysis

The effect of this connected component based filtering, over a small region is shown in Fig- 8 .

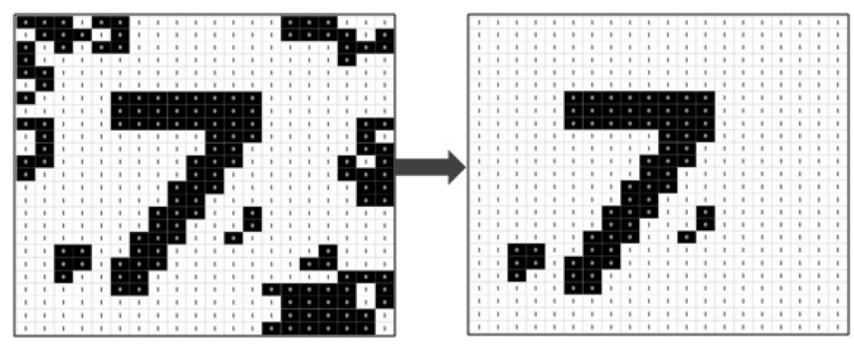

Figure 8. Effect of 8-neighbor connected component analysis

This 8 neighbour connected component analysis based filtering helps in getting a significantly noiseless image $D$ (Fig-9).

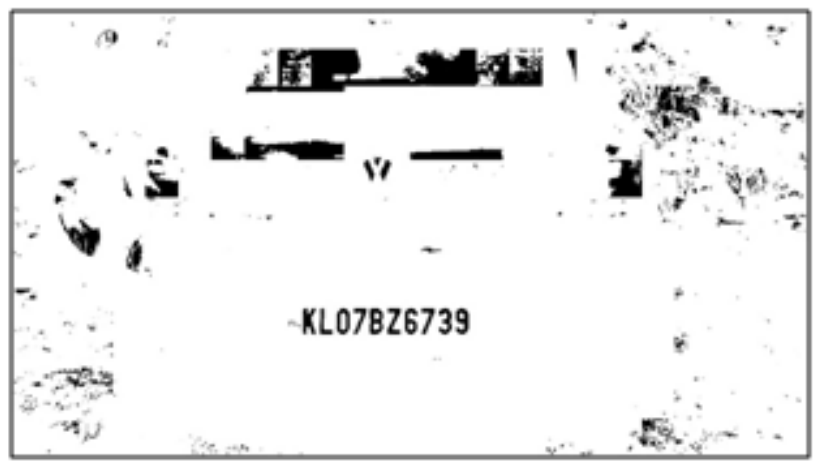

Figure 9. Connected Component based filtered image D of input image C

\subsection{Horizontal Black Run-length based Image Filtering}

Though connected component analysis based filter removes significant amount of noise, some noises which are disconnected from the image border are generally not removed. Connected component analysis based filters starts from the border pixels and eliminates all pixels connected to the border pixels. This leaves some connected components which are present towards the center of the image which are disconnected from border pixels to be retained although they do not pertain to be number plate components. To remove such noises yet another filtering approach is proposed which performs at each row level. 


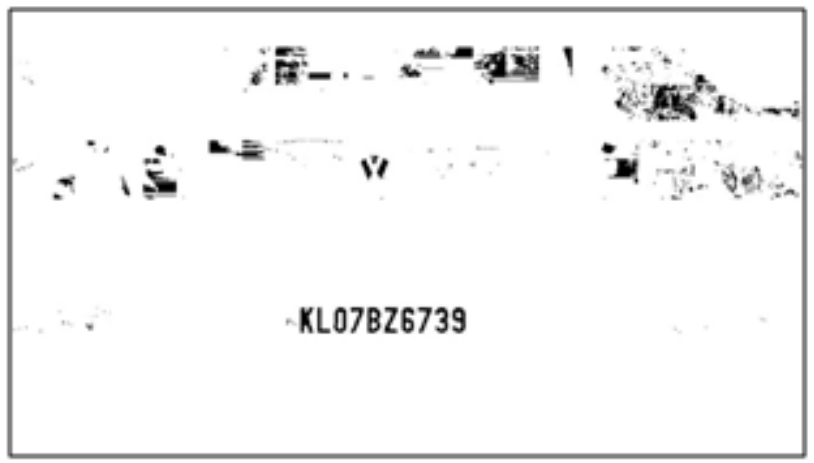

Figure 10. Horizontal Run-length based Image Filtering E of input image D

Each row is scanned for continuous connected black pixels. A single row may have more than one black connected component. If the pixel count of these black run-length components counts is greater than $3 \%$ of the image width $(\mathrm{V})$ then corresponding black pixels are converted to white. Also again we check the total black pixel count for each row, if the count gets less than the 5\% or greater than the $80 \%$ of the image width of $E$ then the entire row is made white.

A survey was carried out within our dataset and it is found that the width of any character present in the number plate is less than the $3 \%$ of the width of the image for resolution $1456 \times 2592$. This hypothesis helped us to get images with significantly less noise. On the other hand, if we observe that a row contains black pixels less than $5 \%$ or greater than $80 \%$ of the width of an image then we conclude that either the row doesn't have any part of the number plate or the row is full of noise. The effect of this image filtering technique has been furnished in Fig-10.

\subsection{Number plate region extraction}

While analyzing the pre-processed image E, it was clearly observed that the image can be segmented into a number of sub-images. The sub-images can be further analyzed to check the presence of the number plate. Along with number plate, sub-images may contain some additional noise components. Horizontal projection profile is used to segment the entire image into subimages for further analysis. The horizontal projection profile for image $\mathrm{E}$ is shown in Fig- 10.

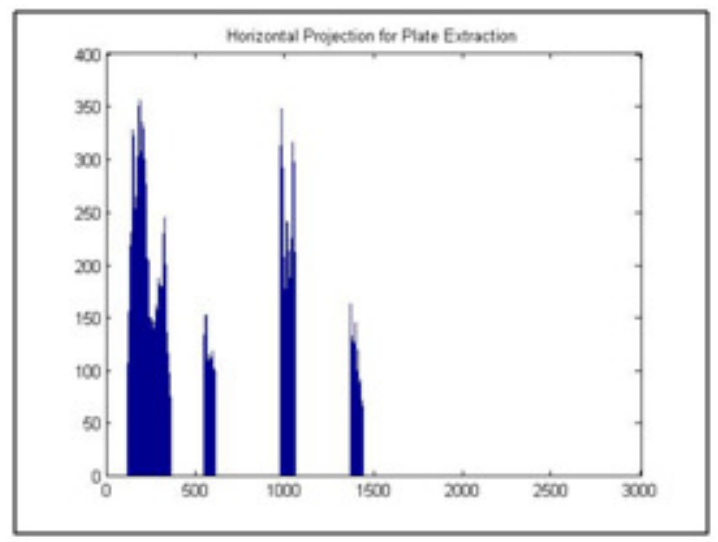

Figure 11. Horizontal Black Profile Projection of E 
From the plot shown in Fig.11, if we find out the drastic shifts from zero and a drastic drop to zero, with the rise and a drop forming one region, in the above profile, we can see four subimages. The number plate can be present in any of these four regions. Sometime, if the number plate runs over two lines, then part of the number plate can be present in two separate subimages, which are adjacent to each other. The other sub-images can be discarded as irrelevant. We have to crop these regions from the image $\mathrm{E}$ by inspecting the projection profile. From our experimental observation, sub-images that correspond to noise or irrelevant regions tend to have lesser black pixels density than sub-images that correspond(s) to number plate region.

First we calculate the average black pixel density of the image E using equation (1).

$$
M=\frac{\text { Number of black pixels in } E}{\text { Size of } E}
$$

From the horizontal profile the maxima point of each dense segment is computed. If the maxima of any segment is higher or equal to $\mathrm{M}$, i.e, the mean black pixel density of entire image, then the sub-image corresponding to that segment of the projection profile is considered to have number plate. However, we also need to ensure that the width of these sub-images is also acceptable. For this, we must calculate width of each segment in the projection profile. If the width of the segment is less than $2 \%$ of the length (Number of rows) of original image $E$ then the sub-image corresponding to that segment of project profile can be discarded.

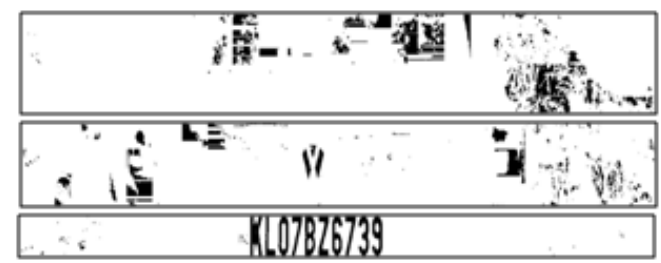

Figure 12. Extracted all probable regions of number plate

\section{Character Segmentation}

From the above sub-images, to segment out the characters we perform two validating criteria explained below.

\subsection{Projection Profile Based Validation}

If the extracted sub-images are observed separately then it can be seen that the characters are separated by a very few white pixels and generally they maintain an equidistance from each other whereas the regions with full of noise do not have such observations. To achieve this, vertical black projection profile has been applied on extracted sub-images to determine the high density black region. One such projection profile is shown in Figure 13.

From the Fig-13, it is very clear that centre peak region represents high density black regions which can be regarded as characters of number plate, if we get black pixel peaks in the alternate segments separated by equidistant segments corresponding to background, until a long stretch of drop to zero is encountered. 


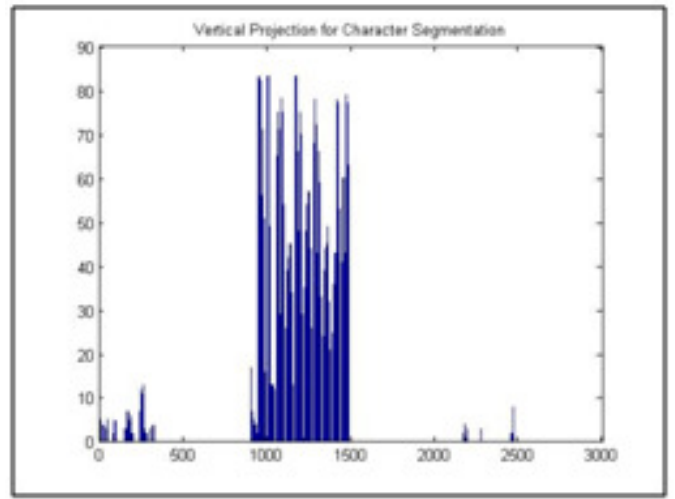

Figure 13. Vertical Black Projection Profile of a sub-image consisting of Number Plate

\subsection{Character Height and Width Validation}

As per our survey on our dataset, it has been observed that if the distance between camera and the car is nearly 10 meters and the resolution of the captured image is 1456x2592 then on an average a valid character on the number plate must contain at least 100 black pixels. Each sub-image is examined separately to discard connected components with size less than 100 pixels. Some of the outcomes of eliminating connected components of size less than 100 pixels for some sub-images are shown in Fig.14.

In addition to the above observation, the size of the characters of the number plate is also uniform in nature. The individual height and width of those character segments will be approximately same. After, eliminating some unwanted smaller components, a minimum bounding rectangle is fit over all remaining connected components.

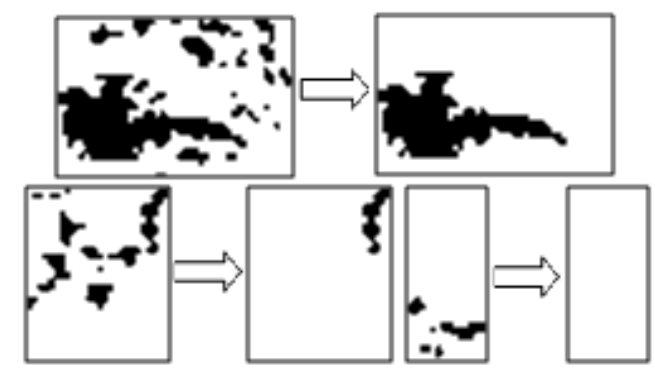

Figure 14. Removal of connected components of smaller size

The minimum bounding rectangles fit around the connected components corresponding to the characters belonging to number plate region, will approximately have the same height and width. It can also be observed that the, begin and end of these rectangles will be aligned to the same point on the Y-axis. So if there are at least 4 or more rectangles which are adjacent to each other and have the properties explained above, then these rectangles can be selected as relevant. We choose 4 rectangles due to the reason; the number plate characters may be spread in 2 lines, with a maximum of four characters in each line. Another criteria, that is employed to check if the segmented characters belong to the number plate is by comparing if the region of these characters match with the region which correspond to the dense projection profile generated in the previous stage. 
Using the above two validation criteria, the character and the number plate regions are segmented and separated from the other irrelevant components as shown in Fig. 15. This segmented region along with the segmented characters is then passed to the character recognition stage.

\section{KL07BZ6739}

Figure 15. Segments after Employing Height-Width Approximation method

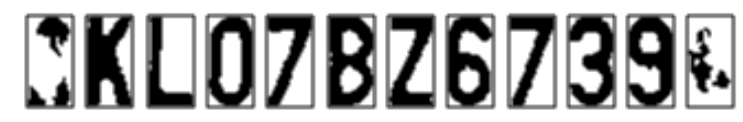

(a)

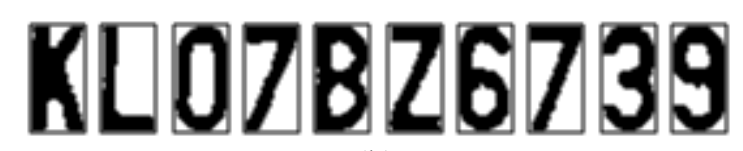

(b)

Figure 16. Segments after Employing White Pixel Ratio over Black Pixel

In order to make sure that the classification stage receives only characters (Alphabets and numerals as inputs), white to black pixel ratio for each connected component enclosed in the minimum bounding rectangle is calculated. If the character enclosed in the rectangle has a ratio greater than 4 (refers to segment maximum white) or equal to 0 (refers to segment full black) then the segment is discarded. This technique produces noise free character segments only. An example of such case and the result after the elimination of the noisy segments is shown in Fig.16 (a) and (b). The non-alphabetical characters enclosed in minimum bounding rectangles are just simulated noise. If the total number of characters segmented is less than 4 , it is discarded from next stage of recognition and classification. The segmented characters are normalized to a new scale of dimension $(40 \times 20)$ before sent for training and classification.

\section{OPTICAL CHARACTER RECOGNITION}

In this stage, Support Vector Machine (SVM) was used for supervised learning of 36 classes (26 alphabets of upper case and 10 digits).During dataset generation, it was observed that number plate generally contains alphabets in upper case only so the same data for lower case alphabets are not considered.

For character recognition, the entire character enclosed by a minimum bounding rectangle is used for training. The image is vectorised by re-arranging the pixel values into one dimensional vector $\mathrm{K}$ of size, $1 \times 800$. Let $\mathrm{F}$ represents total number of vectors. These FxK feature vectors are submitted to SVM for training. The segmented characters from the number plate are then classified using the trained classifier, corresponding ASCII values are written in a text file to achieve complete OCR.

\section{DATABASE}

We have tested our proposed algorithm on 560 different images of license plates. As earlier mentioned, all images are captured at a distance nearly 10meters from the vehicle. The resolution 
of all captured images is $1456 \times 2592$ and they are saved in standard JPEG format. Apart from this database, to establish the proposed method as resolution invariant we have scaled down and scaled up the images in different resolutions and performed the same operation. Detailed discussion is given in the next section.

\section{EXPERIMENTAL RESULTS AND COMPARISON STUDY}

To test the resolution invariant feature 100 images have been scaled down and scaled up in different resolution and employed with the proposed method. It has been observed that the performance of proposed method was accurate in extracting number plates and character segmentation for various sizes of images shown in Table-1.

\begin{tabular}{|c|}
\hline Various image scales \\
\hline $508 \times 904 ; 564 \times 1004 ; 627 \times 1116 ; 697 \times 1240 ;$ \\
$774 \times 1378 ; 860 \times 1531 ; 955 \times 1701 ; 1061 \times 1890 ;$ \\
$1179 \times 2100 ; 1310 \times 2333 ; 1602 \times 2851 ;$ \\
$1762 \times 3136 ; 1938 \times 3450 ; 2132 \times 3795 ; 2345 \times 4175$ \\
\hline
\end{tabular}

Table1: Different image sizes on which the number plate extraction and character segmentation was tested.

The overall performance of the proposed method compared to other methods with respect to number plate extraction, character recognition is shown in Table-2, and Table-3. The accuracy of character segmentation is $95.4 \%$.

It is to be mentioned that the proposed approach is capable of recognizing double lined number plate. An example of such example has been shown in Fig-21.

As the number plate extraction algorithm extracts all the probable number plate regions, so it is possible to recognize multiple number plates from a single image or a double line number plate. An Example of such case is given below in Fig-22 using a synthetic dataset.

Table 2: Performance comparison of the proposed system for number plate extraction

\begin{tabular}{|c|c|}
\hline Methods & $\begin{array}{c}\text { Number Plate } \\
\text { Extraction (\%) }\end{array}$ \\
\hline Lee et al. [26] & 94.4 \\
\hline Chiou et al. [27] & 96.2 \\
\hline Shi et al. [28] & 96.5 \\
\hline Wang et al. [29] & 98 \\
\hline Chang et al. [30] & 98 \\
\hline Deb et al. [31] & 92.4 \\
\hline Jia et al. [32] & 95.6 \\
\hline Kim et al. [33] & 93.5 \\
\hline Duan et al. [34] & 93.6 \\
\hline Roy et al. [35] & 91.59 \\
\hline Proposed & 97.1 \\
\hline
\end{tabular}


Table 3: Performance comparison of the proposed system for number plate recognition

\begin{tabular}{|c|c|}
\hline Methods & OCR Rate (\%) \\
\hline Lee et al. [26] & 95.7 \\
\hline Shi et al. [28] & 89.1 \\
\hline Chang et al. [30] & 94.2 \\
\hline Proposed & 95.72 \\
\hline
\end{tabular}

\section{DISCUSSION AND CONCLUSION}

The above proposed system performs efficiently for wide variations in illumination conditions and different types of number plates. It has the features like double line and multiple number plate recognition. Though there are certain restrictions in this system like- different font style (e.g. italics) and colors of the number plate, excessive skewed number plate, which we consider for our future work.

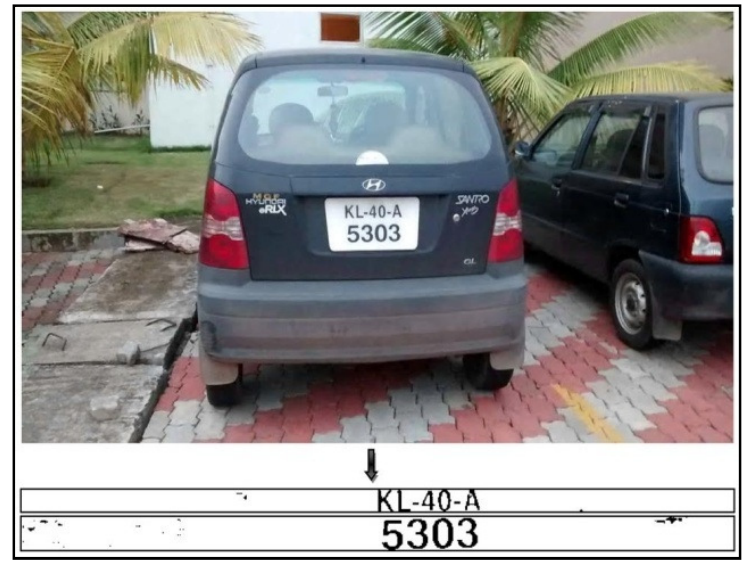

Figure 17. Successful Extraction of Double Lined Number Plate

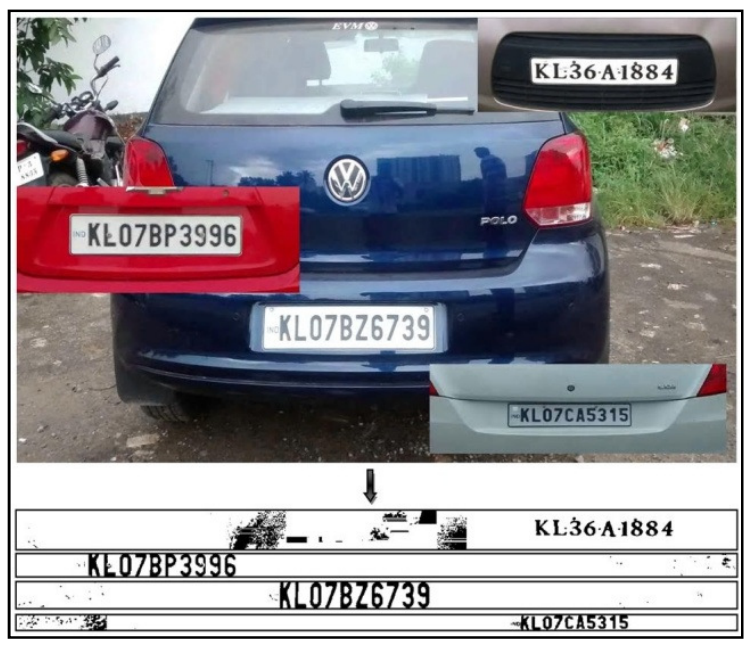

Figure 18. Detection of Multiple Number Plates from Single Input Image 


\section{REFERENCES}

[1] D.R. Salter, 1984, The potential of automatic vehicle identification, International conference on road traffic data collection, Institution of Electrical Engineers, London, England.

[2] K.W.Dickinson, R.C. Waterfall, 1984, Video image processing for monitoring road traffic, International conference on road traffic data collection, Institution of Electrical Engineers, London, England.

[3] I.E.Anagnostopoulos, I.D.Psoroulas,V.Loumos,E. Kayafas, 2008, License plate recognition from still images and video sequences: a survey, IEEE Transactions on Intelligent Transportation Systems, Vol.9, Issue. 3,pp377 - 391.

[4] K.V. Suresh, G. Mahesh Kumar and A.N. Rajagopalan, 2007, Super-resolution of license plates in real traffic videos, IEEE Transactions on Intelligent Transportation Systems, Vol. 8, Issue. 2, pp321 -331 .

[5] A.Clemens, F. Limberger,H. Bischof, 2007, Real-time license plate recognition on an embedded dspplatform, IEEE Transaction on Intelligent Transportation Systems, vol. 1, Issue. 4, pp 34-54.

[6] V. Shapiro, G. Gluhchev, D. Dimov, 2006, Towards a multinational car license plate recognition system, Machine Vision and Applications. Volume 17, Issue 3, pp 173-183.

[7] S. Du, M. Ibrahim, M. Shehata, W Badawy,2013, Automatic license plate recognition (ALPR): a state-of-the-art review, IEEE Transactions on Circuits and Systems for Video Technology, Volume:23, Issue: 2, pp 311-325.

[8] S. Chang, L. Chen , Y. Chung and S. Chen, 2004, Automatic license plate recognition, IEEE Transactions on Intelligent Transportation Systems, Volume. 5, Issue. 1, pp 42 -53.

[9] R. Azad, H. R Shayegh, 2013, New method for optimization of license plate recognition system with use of edge detection and connected component, Third International Conference on Computer and Knowledge Engineering (ICCKE), pp $21-25$.

[10] G.-S. Hsu, J.-C. Chen, and Y.-Z. Chung, 2013, Application-oriented license plate recognition, IEEE Transactions on Vehicular Technology, Volume. 62, Issue. 2, pp. 552-561.

[11] M.D. Chaudhary, J.B. Chinchore, 2014, Towards multiple license plate localization in Indian conditions: an edge density based approach, International Conference on Control, Instrumentation, Communication and Computational Technologies (ICCICCT), pp. $60-65$.

[12] B. Li, B. Tian, Y. Li, D. Wen, 2013, Component-based license plate detection using conditional random field model, IEEE Transactions on Intelligent Transportation Systems, Volume. 14, Issue. 4, pp. 1690-1699.

[13] J. Martínez-Carballido, R. Alfonso-López, J.M. Ramírez-Cortés, 2011, License plate digit recognition using $7 \times 5$ binary templates at an outdoor parking lot entrance, 21 st International Conference on Electrical Communications and Computers (CONIELECOMP), pp. 18 - 21.

[14] M. Shiying, Z. Weixin, L. Na'na, S.Yaxin, H. Wen, 2011, Algorithm for multinational license plate localization and character segmentation, 10th International Conference on Electronic Measurement \& Instruments (ICEMI) (Volume:3), pp. 85 - 89. 
[15] Y. Wen, Y. Lu, J. Yan, Z. Zhou, K.M. Von-Deneen, P. Shi, 2011, An algorithm for license plate recognition applied to intelligent transportation system, IEEE Transactions on Intelligent Transportation Systems, Volume. 12, Issue. 3, pp. 830 - 845.

[16] P. Comelli, P. Ferragina, M.N. Granieri, F. Stabile, 1995, Optical recognition of motor vehicle license plates, IEEE Transactions on Vehicular Technology, Volume. 44. Issue. 4, pp. 790 - 799.

[17] J.K. Chang, 2010, Real-time vehicle license plate recognition on road images from various cameras, Third International Conference on Human-Centric Computing (HumanCom), pp. 1- 6.

[18] A. Wang, X. Liu, 2012, Vehicle license plate location based on improved roberts operator and mathematical morphology, Second International Conference on Instrumentation \& Measurement, Computer, Communication and Control, pp. 995 - 998.

[19] T Naito, T Tsukada, K Yamada, K Kozuka, S Yamamoto, 2000, Robust license-plate recognition method for passing vehicles under outside environment, IEEETransactions on Vehicular Technology, Volume. 49, Issue. 6, pp. $2309-2319$.

[20] J. Jiao, Q. Ye, Q. Huang, 2009, A configurable method for multi-style license plate recognition, Pattern Recognition 42, pp. 358-369.

[21] N Thome, A. Vacavant, L. Robinault, S. Miguet, 2011, A cognitive and video-based approach for multinational license plate recognition, Machine Vision and Applications, Volume 22, pp 389-407.

[22] J.M Guo, Y.F. Liu, 2008, License plate localization and character segmentation with feedback selflearning and hybrid binarization techniques, IEEE Transactions on Vehicular Technology, Volume. 57, Issue. 3, pp. $1417-1424$.

[23] A.M. Al-Ghaili, S. Mashohor, A.R. Ramli, A. Ismail, 2013, Vertical-edge-based car-license-plate detection method, IEEE Transactions on Vehicular Technology, Volume. 62, Issue. 1, pp. 26 - 38.

[24] T. Sirithinaphong, K. Chamnongthai, 1999,Recognition of car license plate for automatic parking system, Fifth International Symposium on Signal Processing and its Applications (ISSPA), pp. 455 457.

[25] J.C.H. Poon, M. Ghadiali, G.M.T. Man, L.M. Sheunp, 1995, A robust vision system for vehicle license plate recognition using grey-scale morphology,Proceedings of the IEEE International Symposium on Industrial Electronics, ISIE '95.

[26] H.-J. Lee, S.-Y. Chen, and S.-Z. Wang, Extraction and recognition of license plates of motorcycles and vehicles on highways, in Proc. Int. Conf. Pattern Recognition, 2004, pp. 356-359.

[27] Y.-C. Chiou, L. W. Lan, C.-M. Tseng, and C.-C. Fan, Optimal locations of license plate recognition to enhance the origin-destination matrix estimation, in Proc. Eastern Asia Soc. Transp. Stu., vol. 8. 2011, pp. 1-14.

[28] X. Shi, W. Zhao, and Y. Shen, Automatic license plate recognition system based on color image processing, Lecture Notes Comput. Sci.,vol. 3483, pp. 1159-1168, 2005.

[29] S. Z. Wang and H. J. Lee, A cascade framework for a real-time statistical plate recognition system, IEEE Trans. Inform. Forensics Security, vol. 2, no. 2, pp. 267-282, Jun. 2007. 
[30] S.-L. Chang, L.-S. Chen, Y.-C. Chung, and S.-W. Chen, Automatic license plate recognition, IEEE Trans. Intell. Transp. Syst., vol. 5, no. 1, pp. 42-53, Mar. 2004.

[31] K. Deb, H.-U. Chae, and K.-H. Jo, Vehicle license plate detection method based on sliding concentric windows and histogram, J.Comput., vol. 4, no. 8, pp. 771-777, 2009.

[32] W. Jia, H. Zhang, X. He, and M. Piccardi, Mean shift for accurate license plate localization, in Proc. IEEE Conf. Intell. Transp. Syst., Sep. 2005, pp. 566-571.

[33] S. K. Kim, D. W. Kim, and H. J. Kim, A recognition of vehicle license plate using a genetic algorithm based segmentation, in Proc. Int. Conf. Image Process., vol. 2. 1996, pp. 661-664.

[34] T. D. Duan, T. L. H. Du, T. V. Phuoc, and N. V. Hoang, Building an automatic vehicle license-plate recognition system, in Proc. Int. Conf. Comput. Sci. RIVF, 2005, pp. 59-63.

[35] A. Roy, D. P. Ghosal, Number Plate Recognition for Use in Different Countries Using an Improved Segmentation, in Proc. 2nd National Conf. on Emerging Trends and Applications in Computer Science, March 2011, pp. 1-5. 